\title{
Conducting polymer composites of polythiophene with natural and synthetic rubbers
}

\author{
Semih Yigit, Jale Hacaloglu, Ural Akbulut, Levent Toppare *,1 \\ Department of Chemistry, Middle East Technical University, 0653I Ankara, Turkey \\ Received 18 January 1995; accepted 6 November 1995
}

\begin{abstract}
Electrochemical synthesis of conducting polymer composites of polythiophene was achieved. Synthetic and natural rubbers were used as the insulating polymer matrices. FT-IR, differential scanning calorimetry (DSC), scanning electron microscopy (SEM) and mass spectrometry (MS) were utilized to characterize the composite blends. The conductivity measurements were done by using a standard four-probe technique. The above-mentioned methods show that the resultant composites have different properties compared to polythiophene due to interaction of the rubbers with electrochemical polymerization of thiophene, whereas the same argument is not valid for the polypyrrole synthesis via the same procedure.
\end{abstract}

Keywords: Polythiophene; Composites; Rubbers

\section{Introduction}

In recent years, conducting polymers with highly extended conjugated electron systems in their backbones have attracted great interest due to their electrical, electrochemical and optical properties [1]. The electrically conducting polymers such as polypyrrole, polythiophene, polyfuran and polyaniline have been examined for possible electronic and chemical applications [2-4]. However, practical applications of the conducting polymers have been slow owing to certain deficiencies like poor mechanical properties and environmental and thermal instability [5-7]. Polythiophene (PTh) has been intensively studied by either electrochemical [8-10] or chemical [11,12] polymerization. Applications of PTh such as electrooptical switching and memory devices [13] or organic batteries [14] have been proposed. The electrochemical polymerization is usually done by electrochemical oxidation of thiophene on a Pt or Pt-coated substrate to obtain a thin uniform film. $\mathrm{Pt}$ is selected because it is inert against the polymerization reaction at the relatively high oxidation potential of thiophene. Electrochemical polymerizations of some composite conducting polymers by making use of an insulating polymer have also been reported [15-17]. The main idea is to obtain homogeneous composites, thus improv-

\footnotetext{
* Corresponding author.

${ }^{1}$ Present address: Department of Chemistry, Bilkent University, 06533 Ankara, Turkey.
}

ing their mechanical properties with little decrease in the conductivities.

In this work, the synthesis and characterization of conducting composites of PTh with a synthetic rubber (SR) and a natural rubber (NR) were studied. Characterization of the composite films was done by using scanning electron microscopy (SEM), differential scanning calorimetry (DSC), FTIR and direct and indirect pyrolysis mass spectrometry (MS) analyses to elucidate possible reactions between rubber and PTh during polymerization.

\section{Experimental}

PTh-SR and PTh-NR composites were prepared by electrochemical polymerization of thiophene onto a synthetic or natural rubber-coated $\mathrm{Pt}$ electrode at a constant potential of $+1.8 \mathrm{~V}$ versus $\mathrm{Ag} / \mathrm{Ag}^{+}\left(10^{-2} \mathrm{M}\right)$ reference electrode. The electrolyses were achieved with a Wenking POS 73 potentiostat.

The insulating rubber films were dip-coated from the toluene solution $\left(10 \mathrm{mg}^{-1}\right.$ ) of a Malezian-based natural ( $\mathrm{mol}$. wt. about 1500000 ) or synthetic (mol. wt. 1000000 ) rubber (cis-1,4-polyisoprene). The amounts of insulating and conducting polymers in the composites were determined gravimetrically.

Polymerizations of thiophene were run in a three-electrode system under controlled potential conditions. The electrolysis 
cell was equipped with $\mathrm{Pt}$ foils $\left(1.5 \mathrm{~cm}^{2}\right)$, as working and counter electrodes, and a capillary $\mathrm{Ag} / \mathrm{Ag}^{+}$as the reference electrode. Acetonitrile (Aldrich Co. 27, 071-7) was used as the solvent; tetrabutylammonium tetrafluoroborate (TBAFB, Aldrich Co. 21, 7966-4) was used as the electrolyte. The natural or synthetic rubber-coated Pt electrodes were also electrolyzed in the same medium in the absence of thiophene in order to check whether there is a change in the structure or in the weight of the insulating polymer.

Conductivity measurements were done by a four-probe technique. The composites were characterized by FT-IR (Nicolet 510 FT spectrometer), DSC (TA Instruments thermal analyst 2000 system) and SEM (IEOL ISM-6400 scanning microscope).

Direct or indirect pyrolysis analyses were achieved by MS. Direct pyrolysis consists of an insertion pyrolysis probe which is our own design, a Balzers QMG 311 quadruple mass spectrometer and a personal computer for the control of the instrument and data acquisition and processing [18]. In the case of indirect pyrolysis, the evolved gases in the pyrolysis chamber were analyzed.

\section{Results and discussion}

The electrooxidation of thiophene (Th) using an SR-and NR-coated Pt anode gives black films which can be peeled off from the Pt electrode surface. During electrolysis, the color of the PTh film turns from green to black as the doping state increases. As soon as the electrolyses are started in the presence of Th, both NR and SR leave the electrode to a certain extent as a viscous material. Thus, the gravimetrically determined composition values can only indicate the upper limit of rubber content in the composites. This behavior is not observed in the presence of pyrrole or in the absence of either Th or pyrrole, which suggests that PTh growth on the

Table 1

Conductivities of SR-PTh films

\begin{tabular}{ll}
\hline$\%$ PTh & Conductivity $\left(\mathrm{S} \mathrm{cm}^{1}\right)$ \\
\hline 65 & $1 \times 10^{-1}$ \\
75 & $2 \times 10^{-1}$ \\
80 & $2.6 \times 10^{-1}$ \\
85 & $3 \times 10^{-1}$ \\
90 & $4 \times 10^{-1}$ \\
95 & $6 \times 10^{-1}$ \\
\hline
\end{tabular}

Table 2

Conductivities of NR-PTh films

\begin{tabular}{ll}
\hline$\%$ PTh & Conductivity $\left(\mathrm{S} \mathrm{cm}^{-1}\right)$ \\
\hline 80 & $3.0 \times 10^{-1}$ \\
85 & $3.5 \times 10^{-1}$ \\
90 & $3.2 \times 10^{-1}$ \\
95 & $3.7 \times 10^{-1}$ \\
\hline
\end{tabular}

Pt surface causes degradation of the rubbers via a possible chemical reaction between PTh and rubber.

Polymeric composite films are obtained by peeling them off from the electrode surface. The films are stored in dry $\mathrm{N}_{2}$ atmosphere because the conductivity of the PTh films is reduced with moisture in air. The measured conductivities according to the weight percent of the PTh in composite blends (with NR and SR) are given in Tables 1 and 2. The high percentages of $T h$ in the composites are due to losses in rubber content during Th polymerization. This did not allow us to obtain high rubber content composite films.

When the films are washed with toluene (solvent for NR and SR), no change is measured in the weight, indicating that the films do not dissolve in the solvent. The washing procedure was repeated for several weeks in order to see whether there is a loss in weight, since natural and synthetic rubber
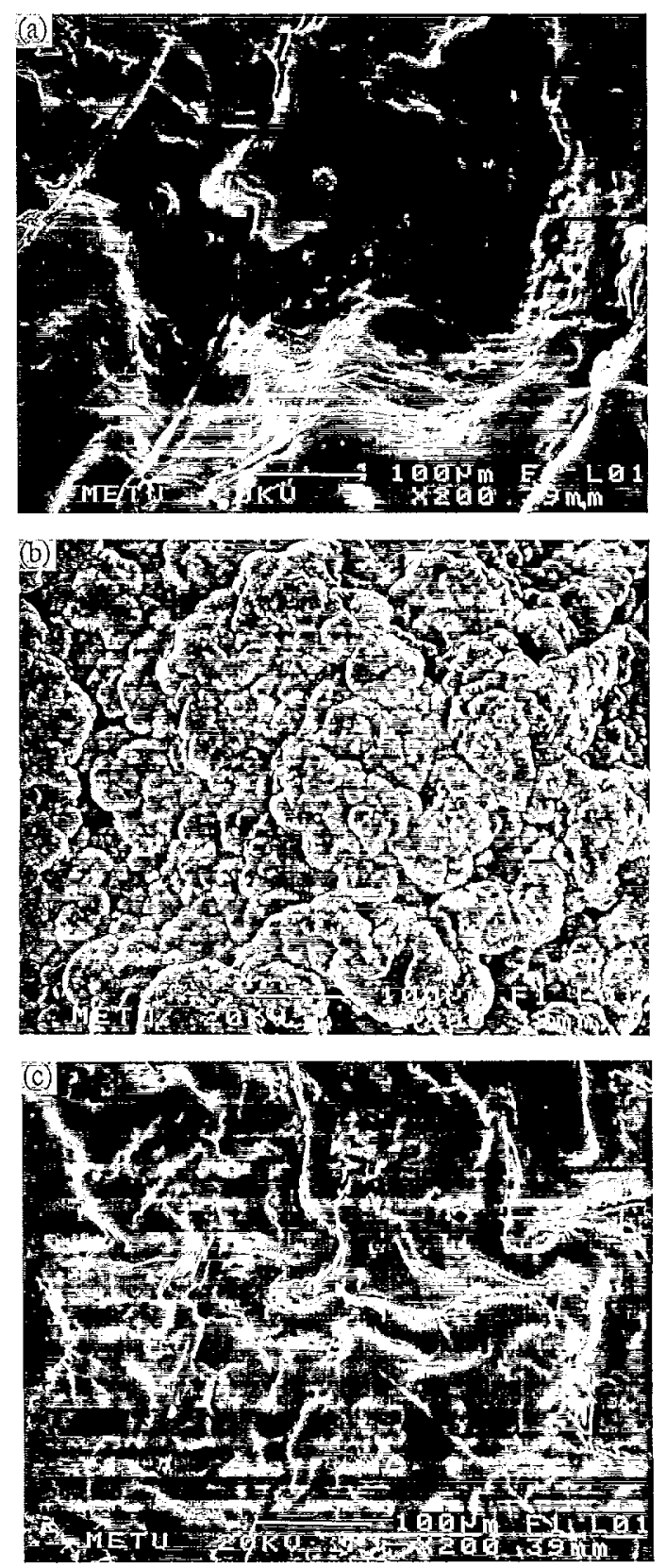

Fig. 1. SEM micrographs of (a) electrode side of composite film (86\% PTh $+14 \%$ NR), (b) solution side of electrolytic film, and (c) washed film. 

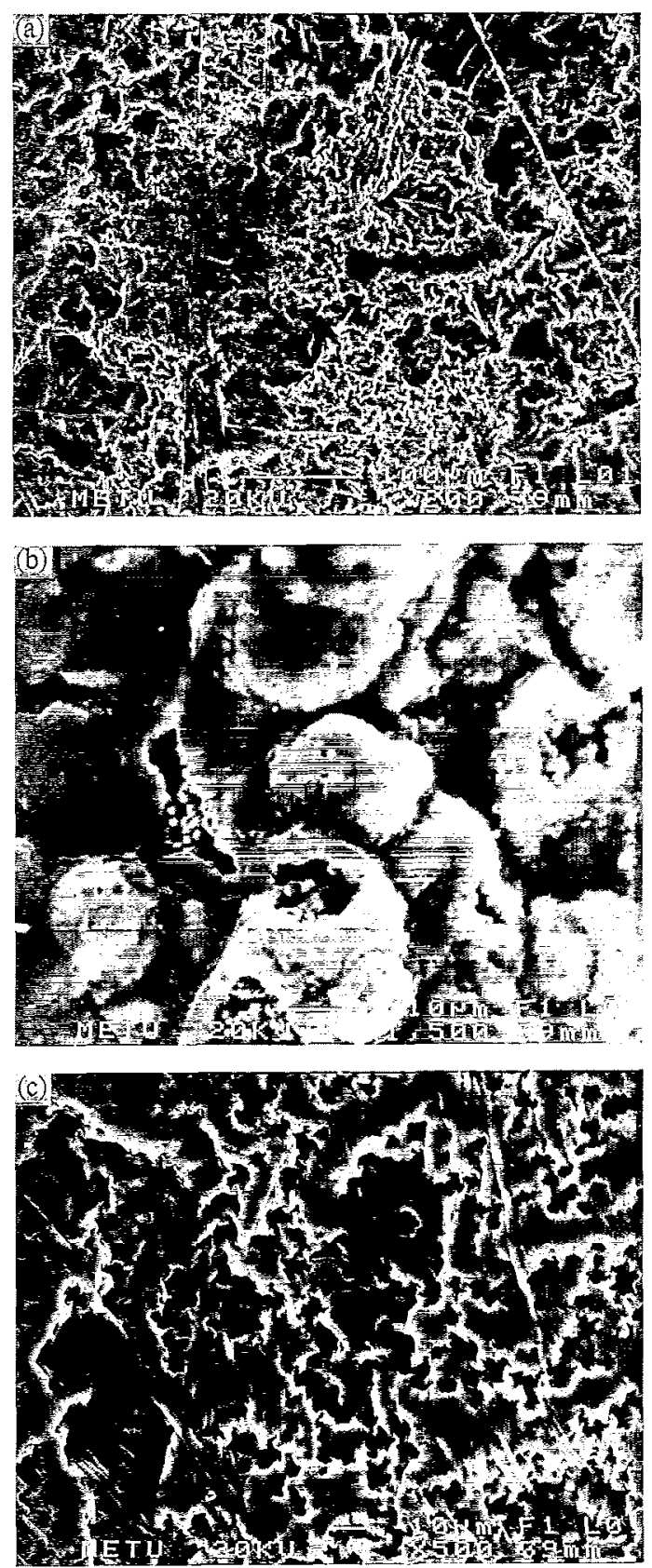

Fig. 2. SEM micrographs of (a) electrode side of composite film ( $62 \%$ PTh $+38 \%$ SR), (b) solution side of composite film, and (c) washed film.

can easily be removed by toluene. No weight losses could be detected and no marked changes were measured in conductivity. This result indicates that a chemical reaction may exist between PTh and rubber. The SEM micrographs of the composite blends show that the PTh grows uniformly in the insulating rubbers. PTh crests grow out of the surface of the coated electrode toward solution producing a cauliflower-like surface. The surface appearances of the films (Figs. 1 and 2) reveal that the electrode and solution sides of the film are quite different, whereas washing after the electrolyses creates no further differences in the surface morphology of the Th film. DSC (Fig. 3) thermograms show that different thermal behaviors exist for NR, SR and the composite films ( $80 \%$ PTh). The glass transition temperatures of NR and SR are around -64 and $-69^{\circ} \mathrm{C}$, respectively. DSC of the PTh-SR composite yields two separate transitions around -70 and $83^{\circ} \mathrm{C}$, which are distinctly different from those of SR (Fig. $3(a))$. This result is further evidence for a chemical interaction between PTh and rubbers.

FT-IR spectra (Figs. 4 and 5) reveal that the rubbers are incorporated into the PTh film. Though some changes in the spectra exist, it is not possible to make any suggestions about the possible chemical interactions between the rubbers and conducting polymer using IR data. To get a better understanding of the interaction, pyrolysis experiments were carried out.

The direct and indirect pyrolysis mass spectra of the composites were compared with those of the corresponding homopolymers. NR and SR show very thermal behavior in accordance with the other thermal analysis data. The principal products from the thermal degradation of these polymers are 1-methylcyclopentene and 1-methylcyclohexene under the experimental conditions of direct pyrolysis [19]. Monomer, dimer, trimer and tetramer peaks are also present in the spectra. The decomposition mainly occurs in a narrow temperature range of 213 to $225{ }^{\circ} \mathrm{C}$. Under the same pyrolysis conditions $\mathrm{PTh}$ starts to degrade at relatively low temperature ranges (about $150^{\circ} \mathrm{C}$ ) by evolution of low molecular weight species such as CS and the monomer [20]. High mass frag-
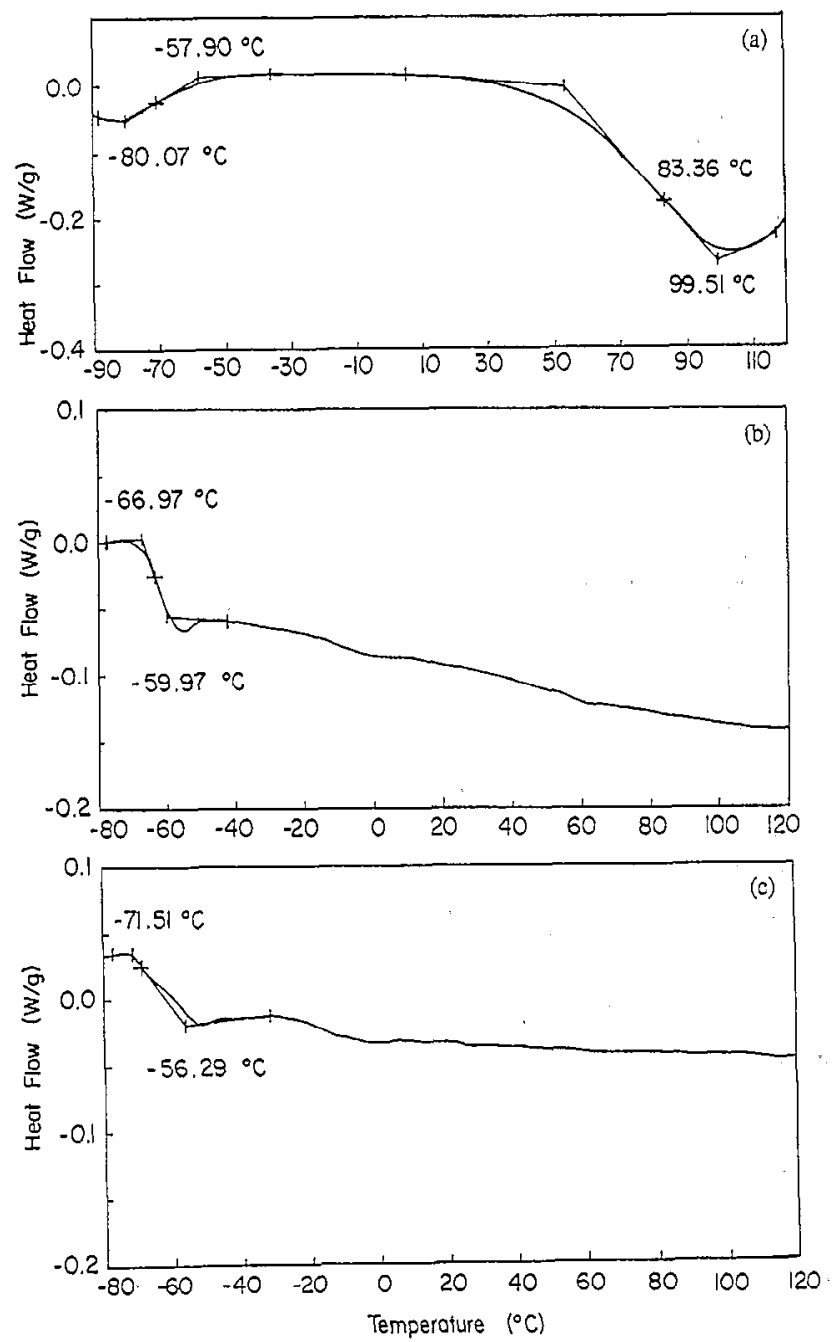

Fig. 3. DSC thermograms of (a) composite film ( $80 \% \mathrm{PTh}+20 \% \mathrm{SR})$, (b) natural rubber (NR), and (c) synthetic rubber (SR). 

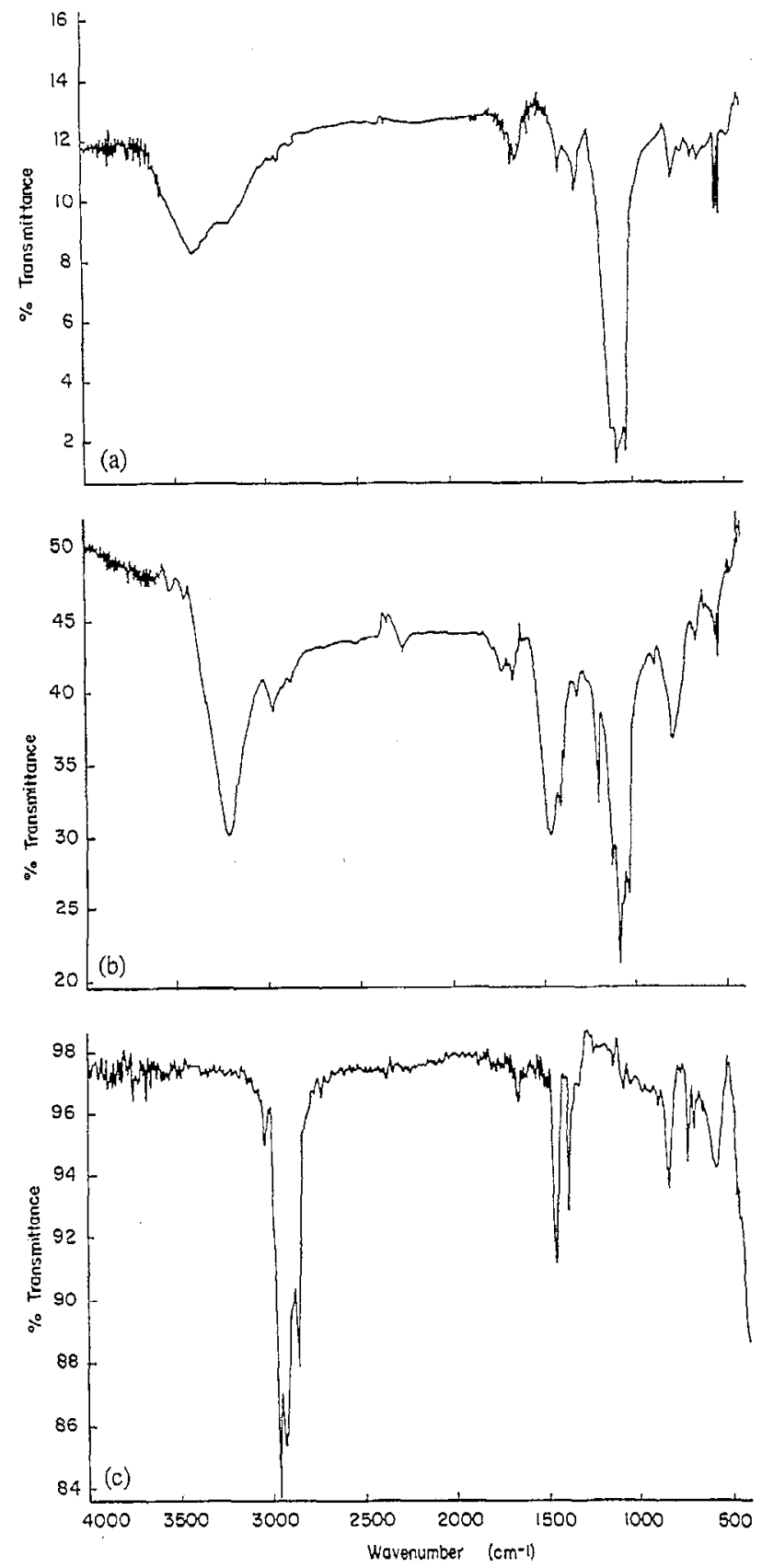

Fig. 4.FT-IR spectra of (a) polythiophene (PTh), (b) composite film ( $80 \%$ PTh $+20 \%$ NR), and (c) natural rubber (NR).

ments are detected above $200^{\circ} \mathrm{C}$. Thermal decomposition of PTh-NR and PTh-SR occurs over a broader temperature range. It is apparent that thermal stability of the composites is lower. The second and the most important difference is disappearance of the peaks related to NR and SR from the pyrolysis spectra. Furthermore, considerable increase in relative yields of ions at $57\left(\mathrm{C}_{4} \mathrm{H}_{9}^{+}\right)$and 174 a.m.u. has been observed:<smiles>C=CCc1ccc(C=C)cc1</smiles>

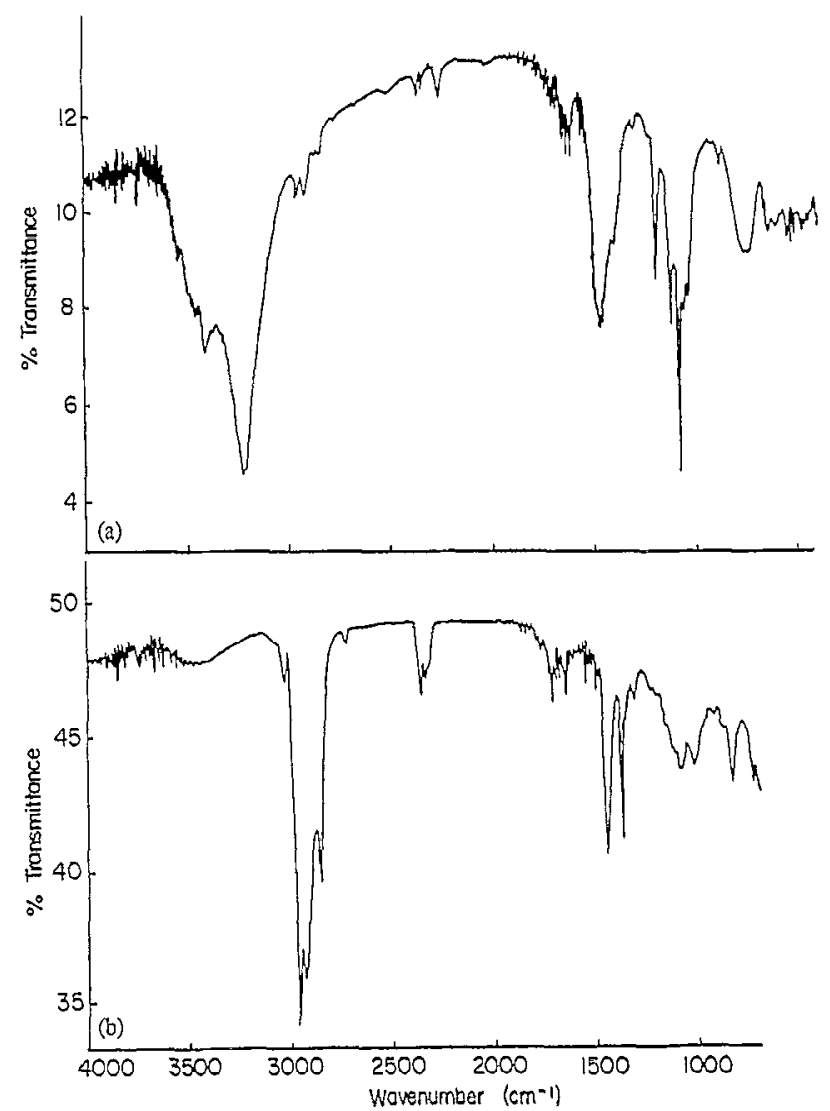

Fig. 5. FT-IR spectra of (a) composite film ( $85 \%$ PTh $+15 \%$ SR), and (b) synthetic rubber (SR).

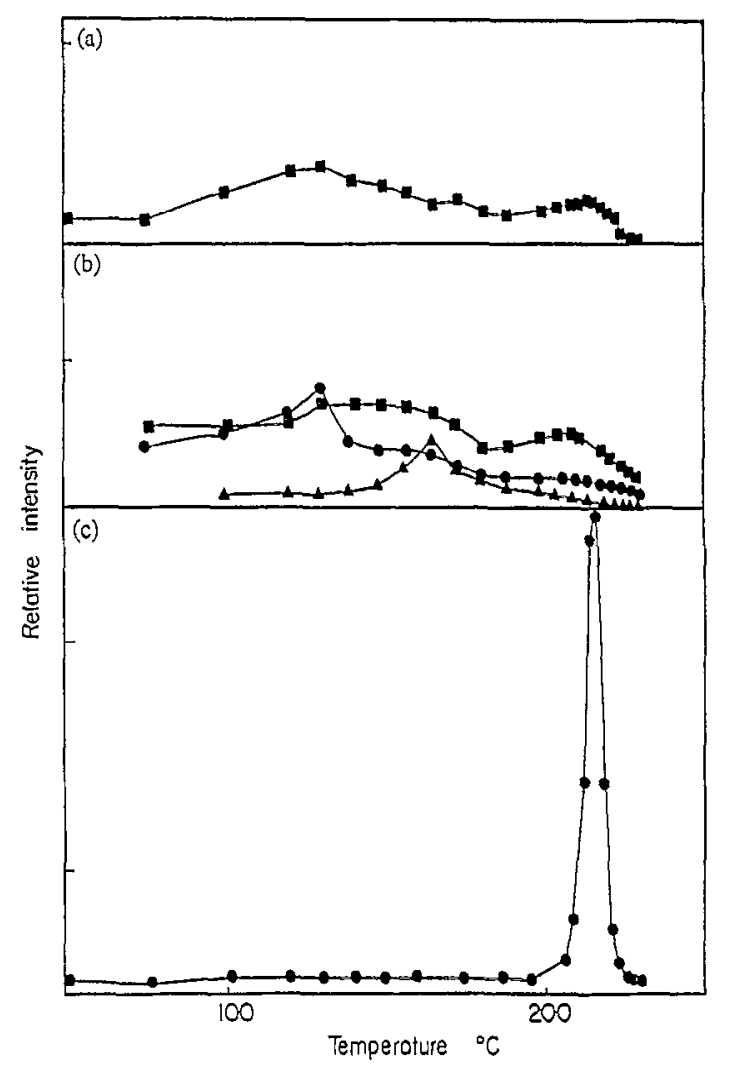

Fig. 6. Ion temperature profiles from (a) PTh, (b) NR-PTh composite film $(82 \%$ PTh $+18 \%$ NR), and (c) NR recorded during direct pyrolysis: $\mathbf{m}, 57$ a.m.u.; 81 a.m.u.; $\Delta, 174$ a.m.u. fragments. 


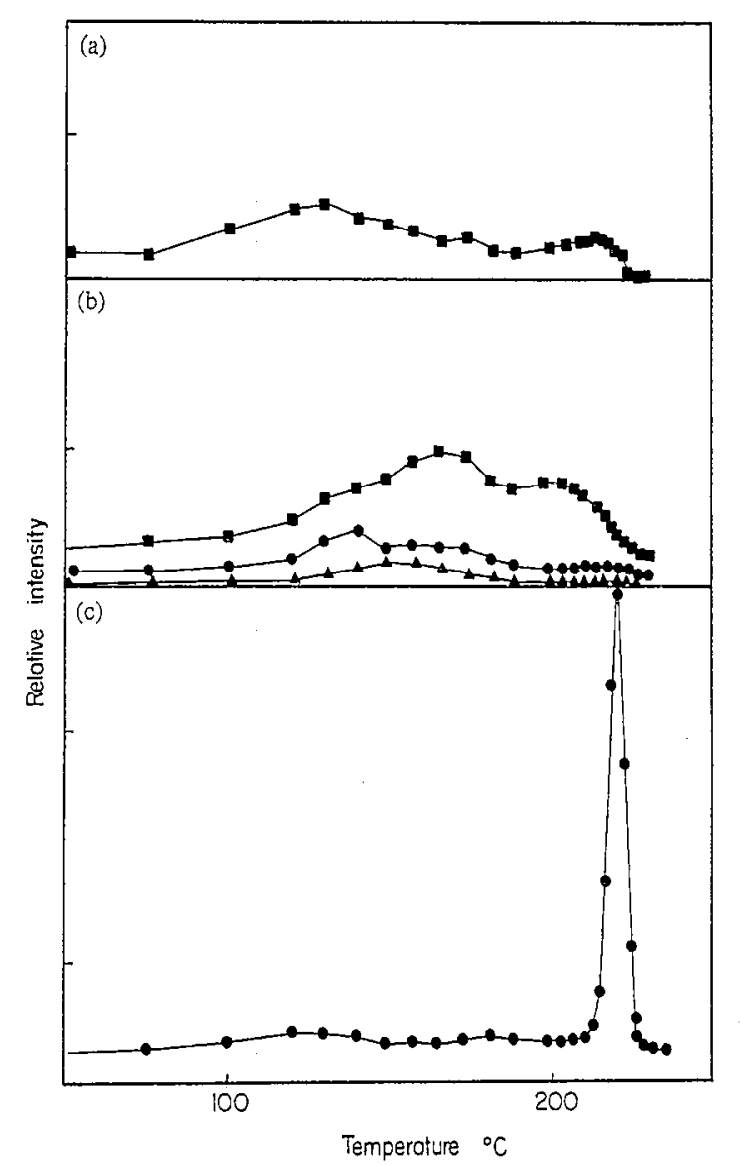

Fig. 7. Ion temperature profiles from (a) PTh, (b) SR-PTh composite film $(77 \%$ PTh $+23 \% \mathrm{SR})$, and (c) SR recorded during direct pyrolysis: $\mathbf{m}, 57$ a.m.u.; $\bullet, 81$ a.m.u.; $\triangle, 174$ a.m.u. fragments.
In Figs. 6 and 7 ion temperature profiles (variation of relative intensities of ions as a function of temperature) of some characteristic fragments detected during thermal decomposition of NR, SR, PTh-NR and PTh-SR composites are given to illustrate the main differences. These results may be directly related to the presence of chemical interaction between the components of the composites. Yet, one can argue that the presence of PTh affects the decomposition of rubber or vice versa. However, other experimental evidence such as degradation of rubber during electrolysis, insolubility of the composites in toluene and thermogravimetric results supports the fact that chemical interaction has taken place.

Indirect pyrolysis (evolved gas) mass spectrometric results confirm the existence of the chemical interaction between the two components of the composites. In this method only the stable thermal decomposition products can be detected. The peaks related to the characteristic degradation products of NR and SR such as 1-methylcyclopentene and 1-methylcyclohexene almost disappear from the pyrolysis mass spectra. It was further noticed that $\mathrm{H}_{2} \mathrm{~S}$ generation is significantly higher in the blends; it starts to appear in the spectra around $200^{\circ} \mathrm{C}, 150^{\circ} \mathrm{C}$ lower than the temperature at which $\mathrm{H}_{2} \mathrm{~S}$ formation is observed from the pure PTh [20] (Fig. 8). Last but not the least, the important point to be discussed is the formation of the $\mathrm{C}_{4} \mathrm{H}_{9}^{+}$fragment at 57 a.m.u. Its formation may be directly related to breaking of the double bonds in NR and SR during polymerization of PTh onto NRand SR-coated electrodes. Based on the results, the following polymerization mechanism is proposed:

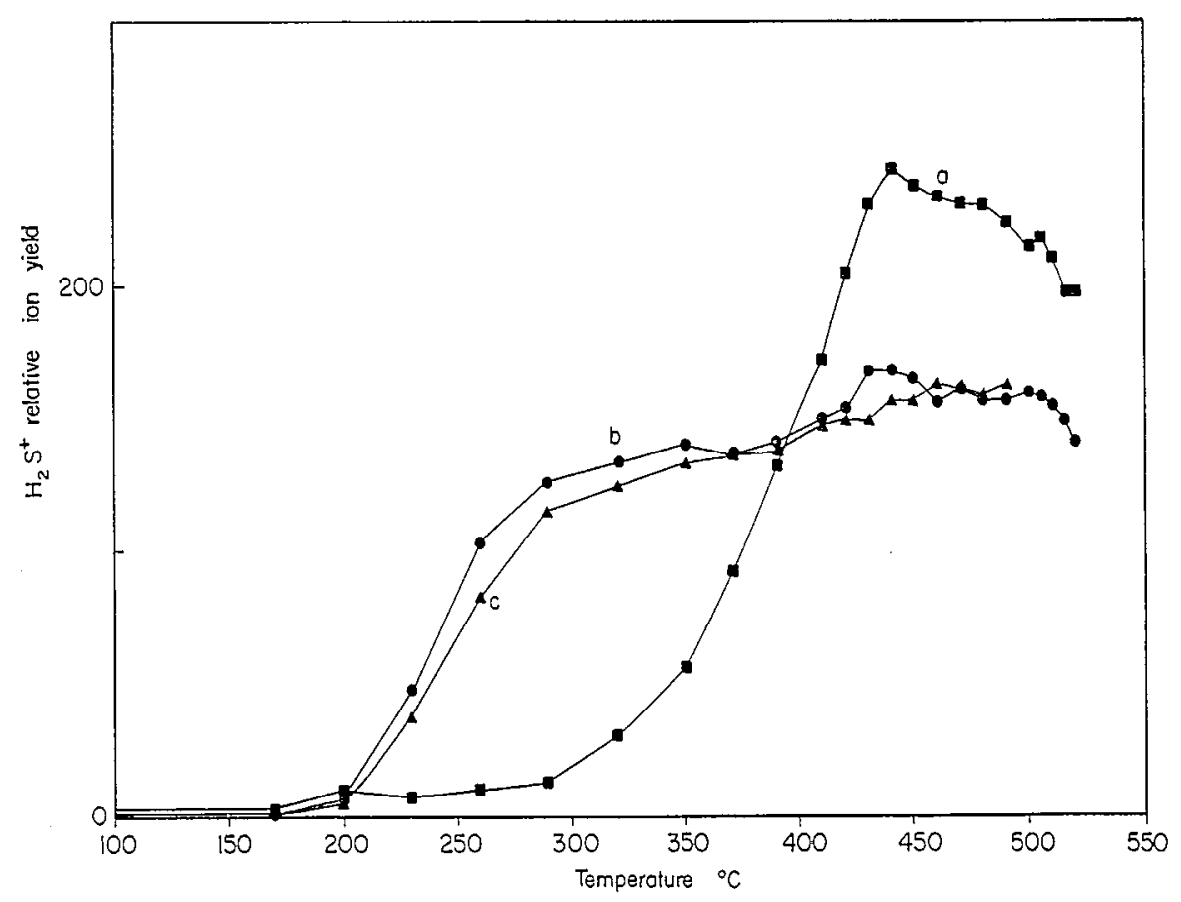

Fig. 8. Ion temperature profiles of $\mathrm{H}_{2} \mathrm{~S}$ from (a) PTh, (b) NR-PTh ( $82 \% \mathrm{PTh}+18 \% \mathrm{NR}$ ), and (c) SR-PTh (77\% PTh $+23 \%$ SR) recorded during indirect pyrolysis. 


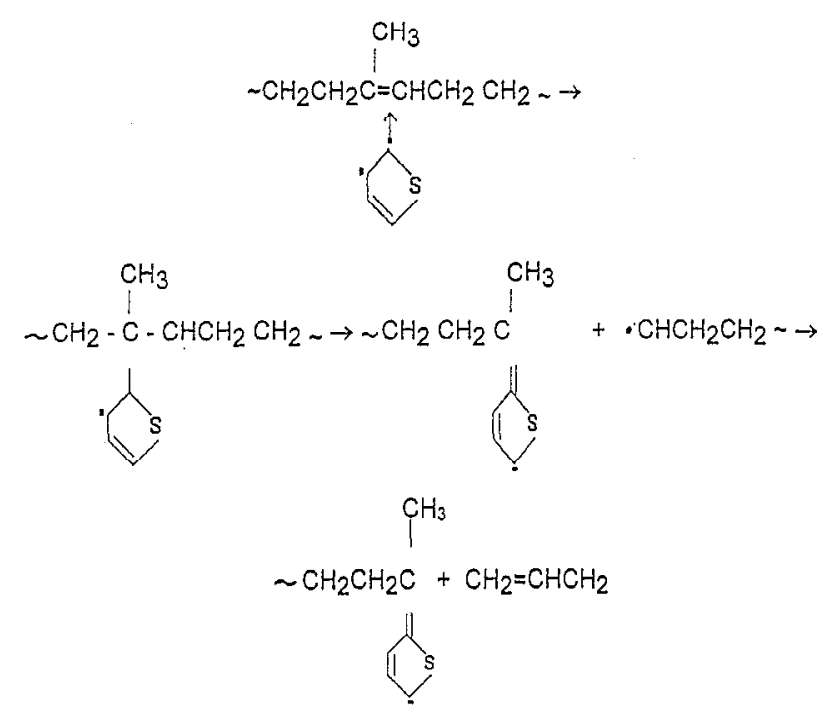

A detailed study of the thermal decomposition mechanism and decomposition products of the composites is in progress.

\section{Conclusions}

In the electrochemical polymerization of Th onto rubbercoated $\mathrm{Pl}$ electrodes, composites of PTh and polyisoprene were formed, together with some chain scission leading to the degradation of the rubbers.

\section{Acknowledgements}

This work is partially supported by the DPT Research Fund (95K-120 498).

\section{References}

[1] T.J. Skotheim (ed.), Handbook of Conducting Polymers, Marcel Dekker, New York, 1986.

[2] A.F. Diaz, K.K. Kanazawa and G.P. Gardini, J. Chem. Soc., Chem. Commun., (1979) 635.

[3] K.K. Kanazawa, A.F. Diaz, W.D. Gill, P.M. Grant, G.B. Street, G.P. Gardini and J.F. Kwak, Synth. Met., I (1979/1980) 329.

[4] G. Tourillon and F. Garnier, J. Electrocmal. Chem. Interfacial Electrochem., 135 (1982) 173.

[5] H. Lindenberger, D. Schafer-Siebert, S. Roth and H. Hanack, Synth. Met., 18 (1987) 37.

[6] M. Zagorcska, A. Proń, S. Lefrant, Z. Kucharski, J. Suwalski and P. Bernier, Synth. Met., 18 (1987) 43.

[7] R. Quian, J. Qiu and D. Shen, Synth. Met., 18 (1987) 13.

[8] D. Braun, D. Moses, C. Zhang and A.J. Heeger, Synth. Met., 55-57 (1993) 4145.

[9] Yen Wei, Guang-Way Jang and Chi-Cheung Chan, J. Polym. Sci., 28 (1990) 219.

[10] S. Hotta, S.D.D.V. Rughooputh, A.J. Heeger and F. Wudl, Macromolecules, 20 (1987) 212.

[11] Z. Cai and C.R. Martin, J. Am. Chem. Soc., LlI (1989) 4143.

[12] T. Yamamoto, K. Sanechika and A. Yamamoto, J. Polym. Sci, Polym. Lett. Ed., 18 (1980) 9.

[13] K. Yoshino, K. Kaneto and Y. Inuishi, Jpn. J. Appl. Phys, 22 (1983) L157.

[14] K. Kaneto, K. Yoshino and Y. Inuishi, Jpn. J. Appl, Phys, 22 (1983) L567.

[15] S. Dogan, U. Akbulut, T. Yalcin, S. Suzer and L. Toppare, Synth. Met., 60 (1983) 27.

[16] H. Lin Wang and J.E. Fernandez, Macromolecules, 26 (1993) 3336.

[17] F. Selampinar, U. Akbulut, T. Yalcin, S. Suzer and L. Toppare, Synth. Met., 62 (1994) 201.

[18] M.M. Fares, J. Hacaloglu and S. Suzer, Eur. Polym. J., 7 (1994) 845.

[19] J. Hacaloglu, M.M. Fares and S. Suzer, Europ, Polym. J., (1994), submitted for publication.

[20] F. Vatansever, U. Akbulut, L. Toppare and J. Hacaloglu, Polymer, (1995), accepted for publication. 